\title{
Interventions via Social Influence for Emergent Suboptimal Restraint Use Felicitas Mokom ${ }^{\mathrm{a}}$ and Ziad Kobti ${ }^{\mathrm{a}}$ \\ ${ }^{a}$ School of Computer Science, University of Windsor, Windsor, Canada, \{mokom, kobti\}@uwindsor.ca
}

KEYWORD

Agent-based simulation

Norm emergence

Suboptimal restraint use

Social influence

\author{
ABSTRACT
}

\begin{abstract}
Although restraint use has increased primarily in developed countries, vehicle accident-related injuries and deaths continue to be a problem. Alongside lack of restraint use, studies involving suboptimal restraint use have gained recent popularity. In this study we investigate the use of social influence for interventions to counter emerging suboptimal restraint use in groups of agents. A multi-agent simulation model is provided where dominant individuals use randomly assigned influence rates to repeatedly alter the knowledge of less influential group members. Cultural influence is implemented via a cultural algorithm and used to simulate individuals affected by beliefs in the community. Objectives include investigating the emergence of patterns of restraint selection and use as well as interventions targeted at more influential agents. Results demonstrate that prominent patterns of behaviour similar to the influential members of the groups do emerge. Furthermore, interventions targeted at influential group members outperform interventions targeted at a percentage of the population at large. Interventions succeed at some level both in the presence and absence of cultural influence.
\end{abstract}

\section{Introduction}

Suboptimal restraint use, which refers to the misuse of restraint systems, is an active interdisciplinary area of research. Motor vehicle accidents are well recognized as a social and economic burden to households and society as a whole. A comprehensive report on road traffic injury prevention released in April 2004 by the World Health Organization (WHO) estimated direct costs associated with roadway collisions at US\$518 billion. In low-income countries, reported costs of US\$65 billion were noted as exceeding amounts received by the countries in aid [PEDEN, 2004]. In a subsequent report on child injury prevention, roadway collisions were identified as the leading cause of death in children aged 15-19 years and the second leading cause of death in children aged 5-14 years worldwide [PEDEN, 2008]. In Canada, although there has been a steep decline in roadway collision-related deaths since 1925 [EBEL, 2003], these accidents remain the dominant cause of injury and death in children below the age of 14 years [HOWARD, 2004].

One of the major contributors to improvements in child road safety is the increasing use of Child Restraint Systems (CRS) [PEDEN, 2004]. It has been reported that in the event of a crash correctly used CRS can reduce fatalities in children by as much as $71 \%$ and serious injury by up to $67 \%$ [HOWARD, 2002]. Unfortunately alongside these accounts are reported rates of CRS misuse ranging from 33\% [SNOWDON, 2009] to $80 \%$ [BRUCKNER, 2009]. Addressing the concern that reports on the transportation of injured children by emergency services have focused on the lack of restraint use while ignoring restraint misuse, a study was conducted to demonstrate the patterns of injury associated with a variety of restraint misuse [BULGER, 2008]. The study can serve as a guide for educational efforts towards appropriate restraint use. Research has shown that suboptimal restrained children are not only injured more often but are also at a higher risk of more serious injuries [BROWN, 2006]. Data collected 
for 152 children aged 2-8 years involved in a collision between 2002 and 2005 identified $82 \%$ of the children as victims of restraint misuse although $94 \%$ were restrained. The alarming rate of misuse may explain the discrepancy between high rates of restraint usage and the persistence of serious injuries or fatalities [BROWN, 2006].

A variety of factors have been noted as contributors to suboptimal restraint use. They include lack of education, parenting style, short travel distance, ease of placement, CRS complexity, social influence and social norms [BLAIR, 2008][BRUCKNER, 2009][BRUCE, 2011]. A parent possessing the correct knowledge for using a CRS for example might choose the suboptimal side location of the vehicle rather than the centre because it is easier or they are pressed for time. Social influence plays a significant role in restraint use and misuse. Individuals tend to adapt their knowledge based on the environment they exist in [KOBTI, 2006]. Studies show that parents often relax rules for optimal restraint use due to social pressures [BRUCKNER, 2009]. Subjective norms or perceived community use has been shown to be a good predictor of booster seat use [BRUCE, 2011]. Specifically, parents' beliefs about booster seat use in their town or community could be used to predict what the parent would do.

In this study we simulate the emergence of suboptimal restraint use as a norm in the presence of social and cultural influence. Furthermore we investigate whether interventions focused on the most influential members of a group or the population at large may assist in the reduction of suboptimal restraint use. Our model is based on a previously implemented model of learning artifact capabilities [MOKOM, 2011]. In the study artifacts were defined as physical objects in the environment that provide some functionality useful to an agent for an objective. Artifact capabilities refer to knowledge acquired by agents for artifact use. Agent-based modelling was used to implement an extended version of a theoretical model for tool capability [ACAY, 2008] that simulated agents learning artifact capabilities individually and socially. According to the tool capability theory an agent is considered to have an artifact or tool capability if the agent knows one way to use the artifact towards one or more of its adopted goals. As in prior work [MOKOM, 2013], we use aspects of the model to simulate a population of drivers or artifact-capable agents, each with the goal of applying and evolving their capability for a restraint. The drivers are organized in hierarchical groups with randomly assigned influence rates determining the degree of influence that group members have over each other. As drivers apply and evolve their restraint capabilities, other members of their group potentially influence them. To simulate the effects of culture we utilize a cultural algorithm to extract prominent patterns of restraint use from the population at large and use them to further influence driver behaviour. One objective is to observe the emergence of suboptimal restraint use as a norm as agents adapt their knowledge. Another objective involves demonstrating the effects on restraint use when agents are influenced socially by other agents or culturally by beliefs in the community as a whole. Finally we wish to explore social influence as a means towards intervention in the reduction of suboptimal restraint use.

The next section provides some background on related work and is followed by our restraint capability model. We then provide details on experiments conducted and results obtained. The final section provides conclusions deduced and future work.

\section{Background}

In our prior work [MOKOM, 2013] we provided a model to study the emergence of restraint misuse within a group of agents in the presence of social and cultural influences. This study is an extension that investigates an additional hybrid form of influence as well as interventions at group and population levels.

\subsection{Restraint Misuse}

The subject of restraint misuse has gained recent popularity particularly in the social sciences. One study relevant to our work involves the use of the theory of planned behaviour as a fabric 
for demonstrating factors most effective at predicting parents' use of booster seats [BRUCE, 2011]. The study concluded that parents' awareness of the benefits was the strongest predictor followed by their perceptions of the use of boosters in their community. The study also cautioned that the relevance of peer influences on parental behaviour should not be neglected.

A variety of intervention strategies have been suggested and investigated. Suggestions have included community-based interventions, legislation, education and targeting risky drivers. Some researchers have suggested the development of culturally sensitive intervention programs arguing that race is a contributing factor to suboptimal use [RANGEL, 2008]. Choice based conjoint analysis was used in simulations to determine the combination of factors most influential in the decision to use booster seats [CUNNINGHAM, 2011]. The results suggested enforcement, injury prevention, advertisements and use of boosters by other parents in the community as interventions. It is a common contention however that no single intervention is perfect and always depends on the targeted population. Closely related to our work is an agent-based cultural model for learning how to apply child safety restraint [KOBTI, 2006]. In the model agents operating in a cultural framework learned two aspects of CRS use collaborating with others in the midst of possible interventions by health care professionals. The two aspects were selection of a CRS and selection of a location in the vehicle for it depending on the age, weight and height of the child. The first distinction with our work is that we consider not only selection of the CRS and its placement within the vehicle but also all the steps that would constitute use of the CRS. For example, proper use of a booster would also involve correctly attaching the booster to the vehicle and the child to the booster. To that effect we contend that while the prior model may be suitable for exploring the lack of restraint use, our model is more appropriate for investigating restraint misuse, which constitutes both proper selection and proper use of restraints [BROWN, 2006][BLAIR, 2008]. Additionally our model examines the effects of social influence in terms of a hierarchy of influential members within groups.

\subsection{Norm Emergence}

Early studies in norm development within a group described the process as gradual and informal [FELDMAN, 1984]. In his study Feldman stated four ways in which norms could emerge: explicit statements by the group leader or other influential members, relevant events in the group's past, the primary behaviour becoming the expected one, and inherited behaviour from past experiences. Norms have been defined as expected behaviours in given situations [OPP, 2001]. A comprehensive summary of the varying agent-based simulation models of norms is provided in [SAVARIMUTHU, 2011].

A study closely related to our work examined norm emergence via social learning [SEN, 2007]. The model involved agents learning from repeated interactions with each other in a gameplaying framework. In every iteration an agent was paired with another randomly selected agent in the group to play against. The study concluded that the interactions resulted in the emergence of norms. In our work we aim to achieve norm emergence via social influence by simulating more influential agents altering the knowledge of less influential agents. To that effect part of our work is an example of the first type of norm emergence stated by Feldman: explicit statements by more influential group members. In addition we simulate the effects of culture by allowing the agent to be influenced by the beliefs of the community, inspired by the suggestion that parents' perception of restraint use in the community affects their restraint use behaviour [BRUCE, 2011].

\section{Restraint Selection and Use Model}

\subsection{Environment Description}

The environment consists of a population of agents organized in groups of varying sizes. 
The idea is to simulate extended families of drivers living within a community. All agents possess some capability for restraint use. Each agent has the same objective: to apply its knowledge for selecting and using a restraint. Prior to applying its knowledge, an agent may adapt its capability as a result of an encounter with another group member or influence from beliefs in the community.

\subsection{Knowledge Structures}

Each agent's knowledge captures what it knows about selecting and using a particular type of restraint. Selection knowledge maintained by an agent is divided into four predefined age ranges obtained from standard knowledge [KOBTI, 2006]. We omit weight and height of the child from the selection process because we believe it should have no significant effect on our model, since our focus is on the emergence of suboptimal use and intervention through social influence. The five possible restraints an agent can select are are "rear facing", "forward facing", "booster", "seatbelt" and "none", represented in binary strings $R_{1}$ to $R_{5}$ respectively. "None" represents the child being unrestrained in the vehicle. If a bit is set to ' 1 ' then the agent considers the respective restraint as a possible selection for the associated age range. An agent's restraint selection knowledge is as follows:

$$
\begin{gathered}
\operatorname{SelKn}(S K)=\{ \\
S K_{1}=\left\langle 00,13,\left[R_{1} R_{2} R_{3} R_{4} R_{5}\right]\right\rangle, \\
S K_{2}=\left\langle 13,49,\left[R_{1} R_{2} R_{3} R_{4} R_{5}\right]\right\rangle, \\
S K_{3}=\left\langle 49,97,\left[R_{1} R_{2} R_{3} R_{4} R_{5}\right]\right\rangle, \\
S K_{4}=\left\langle 97,145,\left[R_{1} R_{2} R_{3} R_{4} R_{5}\right]\right\rangle \\
\}
\end{gathered}
$$

As an example consider an agent that knows that a child aged between 49 and 97 months should be placed either in a booster seat or secured with a seat belt. A partial representation of the agent's selection knowledge would thus be $(49,97,[00110])$.
Restraint use knowledge involves the operations that the agent performs in order to use the restraint. In our model the number of operations is fixed for each type of restraint. In accordance with the artifact capability model [MOKOM, 2011] restraints are artifacts with parts that have attributes. Restraints themselves can have attributes as well. All attributes have a fixed set of possible values representing no use, suboptimal use and optimal use respectively. The use of three fixed values allows simplification and generalization within the model since actual possible values for restraints and their parts vary with each type of restraint. Knowledge for each operation is represented in a binary string as a vector of selected values for each attribute. An agent's restraint use knowledge is as follows:

$$
\begin{aligned}
& U s e K n(U K)=\{ \\
& U K_{1}=\left\langle V_{1} \ldots V_{n}\right\rangle,
\end{aligned}
$$

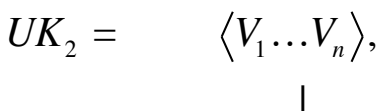

$$
\begin{aligned}
& U K_{k}=\begin{array}{c}
\left\langle V_{1} \ldots V_{n}\right\rangle \\
\}
\end{array}
\end{aligned}
$$

for $n$ total attributes and $k$ operations. For example the operation knowledge (001001) represents a restraint with 3 total attributes with the chosen values $<0,2,1>$. The operation specifies no use for the first attribute, optimal use of the second attribute and suboptimal use for the third.

Belief spaces are used to capture patterns of selection and use within a group of agents or the population at large. At any time these spaces can be probed for the current "belief" of the group or population, that is, the prevalent knowledge that exists. Belief space knowledge is represented as follows:

$$
\begin{gathered}
\text { BeliefSpace }(B)=\{ \\
B\left(S K_{i}\right)=\left\langle P_{1} \ldots P_{s}\right\rangle, i=1 \ldots 4 \\
B\left(U K_{j}\right)=\left\langle Q_{1} \ldots Q_{t}\right\rangle, j=1 \ldots k \\
\}
\end{gathered}
$$


where $s$ (fixed at 32 in the model) is the number of possible patterns used to represent selection knowledge for each of the 4 age ranges. Each $P_{l}$ is a tuple representing a counter and a binary string: $\langle c, x\rangle$ where $x$ ranges from [00000] to [11111] and $c$ represents the number of agents with $x$ as their selection knowledge for an age range. For use knowledge $t$ is the number of possible patterns for each of the $k$ operations. If $r$ is the total number of attributes then $t=2^{r * 2}$. Each $Q_{v}$ is a tuple representing a counter and a binary string: $\langle d, y\rangle$ where $d$ represents the number of agents with the binary string $y$ as their use knowledge for a particular operation.

\subsection{Simulating Social Influence}

The simulation model uses randomly assigned influence rates in order to simulate social influence. These rates could be considered for example as representative of agents' ages, in a community where older agents have influence over younger ones. Influence rates are deemed comparable such that for agent $a$ and agent $b$, if $\inf (a)>\inf (b)$ then agent $a$ has influence over agent $b$ with a degree of influence: $\inf (a)-$ $\inf (b)$. At the start of the simulation each agent is given its own knowledge on selecting and using the same type of restraint. At each simulation step each agent randomly encounters another agent in its group. During the encounter the more influential agent injects its knowledge into the less influential agent. This is accomplished by replicating each bit of the restraint selection knowledge and each attribute value of the restraint use knowledge at a probability of the degree of influence. This allows for more influential agents to propagate their knowledge faster within their respective groups and the population at large and is synonymous to a more influential person having more success at changing the attitudes of another. Our contention is that with time the knowledge possessed by the more influential agents will dominate each group and designate an expected pattern of behaviour. To support the notion that agents will not always accept the new information, agents are also assigned a random retention rate. This rate specifies the chances that an agent will allow itself to be influenced. After each simulation step, each agent contributes its knowledge to its group belief space and the population belief space.

\subsection{Simulating Cultural Influence}

The notion that agents may be affected by perceived use of restraints in their community is captured by simulating cultural influence. Cultural influence is simulated in the model via the use of a cultural algorithm [REYNOLDS, 2004]. In a cultural algorithm selected individuals from a population space contribute knowledge to a global belief space via an acceptance function. Knowledge maintained in the belief space affects the population via an influence function. Different types of knowledge may be maintained in the global belief space including situational, normative, topographic, historical and domain. In our model the belief spaces maintain patterns of restraint selection and use in the form of normative knowledge contributed by all agents in a group for group belief spaces, or all agents in the population for the population belief space. The framework for our cultural algorithm is shown in Figure 1. Adjusting knowledge contributed to belief spaces involves sorting it according to the prominent patterns of selection and use in the group and population respectively.

To simulate the effects of culture, the most prominent pattern in the population belief space is used in each simulation step as an additional influence factor on the population. The idea is to simulate individuals in the population being affected by beliefs in the community in addition to encounters in their respective groups. We do not model any form of influence from the group belief space. Each agent maintains a randomly assigned learning rate as well as a retention rate. Learning rates are comparable in the same manner as influence rates. Any agent that has been affected by an encounter with a more influential agent can be affected culturally. When affected by culture, the most prominent pattern in the population belief space influences the affected agent by injecting each bit of the 
selection knowledge and each attribute value of the use knowledge at the probability of the respective agents' learning rate. The retention rate is used to determine whether the agent allows itself to be influenced.

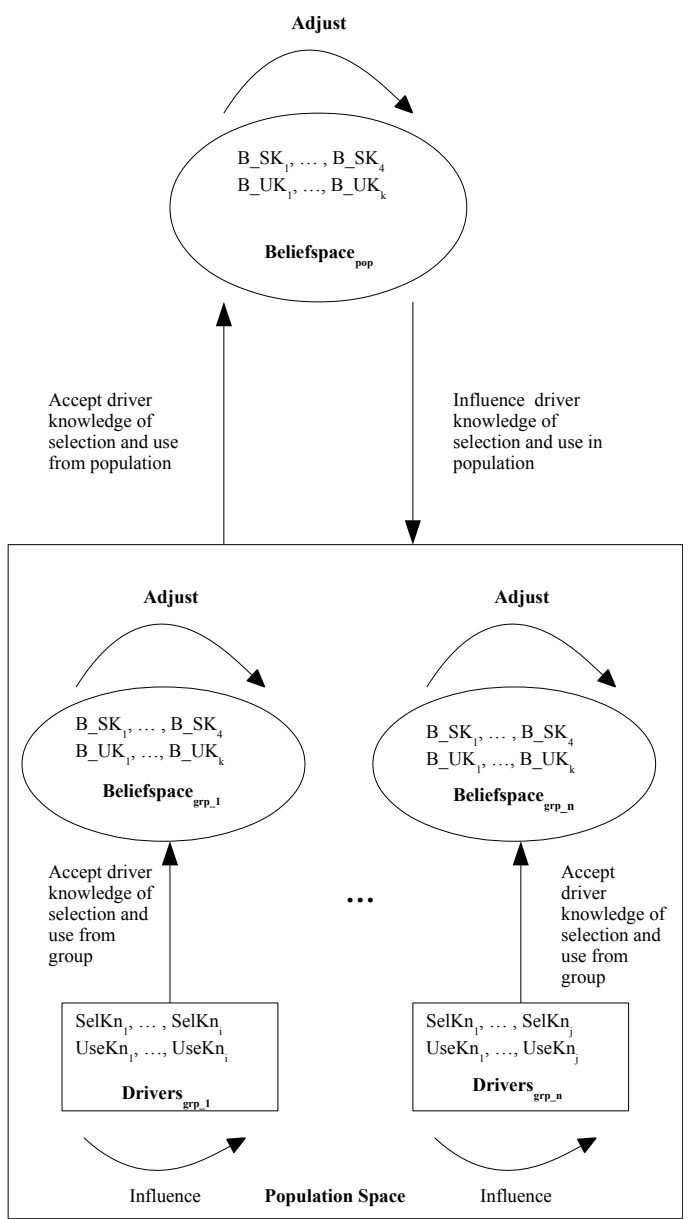

Fig. 1 Cultural algorithm applied to $n$ group of drivers with group level and population level influence for $i$ drivers in group 1 through $j$ drivers in group $n$. Belief spaces maintain restraint selection knowledge for 4 child age ranges and restraint use knowledge for $k$ operations.

\subsection{Intervention Framework}

In the current model interventions are only tested for restraint use knowledge. Two types of interventions are supported. At group level the individual with the highest influence rate is targeted for intervention and provided with the correct restraint use knowledge. The knowledge injection occurs at a probability of the respective agents' learning rate. In the second type of intervention the top $4 \%$ individuals with the highest influence rates in the population are targeted for intervention. Once again, these individuals are injected with the correct restraint use knowledge and accept the knowledge at a probability of their learning rates. The idea is that if experiments demonstrate that the knowledge possessed by the most influential agents can guide the group or population at large towards restraint misuse, then such knowledge can equally be used via interventions to cause an emergence of correct restraint use. The next section provides details on the evaluation process in the determination of the effectiveness of the interventions. Using knowledge resident in the respective belief spaces, agent performance can be measured at group and population levels prior to and after interventions.

\subsection{Performance Measure}

In order to evaluate agent performance before and after interventions are applied, the belief spaces are probed for the restraint use patterns in the group and population respectively. Selected patterns are measured against the predefined standard restraint use knowledge, which is considered the correct knowledge. The objective is to determine whether interventions have a positive effect on the performance of drivers in a group and the population at large. Performance measure of restraint use is computed by matching all attribute value selections of each operation and determining an average score. The score $s$ of an agent's use knowledge $U K$ as specified in Formula 2 is computed as follows:

$$
\begin{aligned}
& f\left(V_{i}\right)=\left\{\begin{array}{cc}
1 & \left|V_{i}-W_{i}\right|=0 \\
0.5 & \left|V_{i}-W_{i}\right|=1 \\
0 & \text { otherwise }
\end{array}\right. \\
& s(U K)=\operatorname{avg}\left(\sum_{i=1}^{n^{* k}} f\left(V_{i}\right)\right)
\end{aligned}
$$


for $n$ total restraint attributes and $k$ operations. $W_{i}$ represents the correct attribute value selection for attribute $i$. This simple evaluation process is facilitated by the fact that the number of possible attribute values in our model is fixed at 3 , specifying no use, suboptimal use and optimal use. A final average is computed for all agents in a group and all agents in the population to determine a score signifying the performance of all respective agents.

\section{Experiments and Results}

In the test environment 50 individuals are randomly organized into 6 groups of varying random sizes [4,12], each group representative of an extended family of drivers. It is our contention that groups of varying sizes gives credibility to results obtained from population level experiments since it provides a more realistic environment. Agents are assigned random influence rates, learning rates, retention rates and selection and use knowledge for one single type of restraint: Booster. Booster is defined as an artifact with 2 artifact attributes: location and attach that specify where the agent places the restraint in the vehicle and how the restraint is attached to the vehicle respectively. Booster also has 2 artifact parts: Shoulder_belt and Lap_belt, each with a single attribute: attach specifying attachment of the respective belt to the vehicle with the child in the restraint. Booster is therefore a restraint with a total of 4 attributes. Possible values for all restraint attributes as explained in the model $\{0,1,2\}$ represent no use, suboptimal use and optimal use. Guidelines on minimal recommendations for appropriate booster use [BULGER, 2008] are used to define 3 operations. The first involves selection of a location within the vehicle to place the restraint, the second involves the attachment of the restraint to the vehicle and the final operation involves the attachment of both belts.

We conduct two series of experiments. In the first series the objective is to determine whether a norm emerges due to social interactions between group members or individuals' beliefs about restraint use in the population. In the second we apply our intervention framework to determine if appropriate injection of correct knowledge into particular individuals can have a positive effect on the society.

\subsection{Norm Emergence Test Cases}

The first test case for norm emergence investigates whether a norm emerges in the presence of social influence at group level. Alteration of an agents' knowledge at each time step is dependent on random encounters with more influential members of its group.

In the second test case norm emergence is investigated at population level in the presence of social, cultural and a hybrid form of influence. For cultural influence agents are affected at each time step by social influence, then additionally by the current most prominent pattern of restraint selection and use in the population belief space. In the hybrid form agents are randomly affected by social influence only or by cultural influence. The hybrid influence tests the scenario where an agent at any given time can decide to be affected by beliefs in its community or not.

\subsection{Intervention Test Cases}

The first intervention test case involves targeting the individual with the highest influence rate in each group and injecting that individual with the correct restraint use knowledge.

In the second intervention test case the top $4 \%$ agents with the highest influence rates in the population at large are injected with the correct restraint use knowledge. Experiments are conducted in the presence of social influence only, cultural influence only and a blend of both. In the social influence only scenario agents are affected by random encounters with other group members. For cultural influence, agents are affected by both random encounters and the most prominent pattern in the population belief space. In the hybrid case, agents are randomly affected by either social influence or cultural influence. All interventions occur at time step 1000 and performance scores 
are obtained using the performance measures in Formula 4.

\section{Results and Discussion}

Results for the norm emergence test cases are shown in Tables 1, 2 and 3. Each result shows emergent patterns from 1 through 5000 steps. The second and third columns of Table 1 show the average distinct selection and use patterns across all groups. The last two columns show the mean of the number of agents using the most dominant patterns in each group across all groups. In Table 2 the second and third columns show the number of distinct selection and use patterns that emerge from the population at large for social influence while the fourth and fifth columns show corresponding results for cultural influence. The results for hybrid influence are shown in Table 3.

The second and third columns of Table 1 demonstrate emergent norms as average distinct patterns of both restraint selection and use decrease over time across all groups. The last two columns show that regardless of varying group sizes the majority of agents in each group converge towards the most dominant pattern in the group. Tables 2 and 3 provide further confirmation of emergent patterns of selection and use in the population at large, as the number of distinct patterns reduced over time for all three types of influences. Patterns of restraint use seemed to decrease more in the case of social influence than when cultural influence was involved suggesting a population's resistance to change in the presence of culture [KOBTI, 2006]. Subsequent intervention results are used to demonstrate that these dominant patterns are similar to the patterns of the most influential agents.

Results for the intervention test cases are shown in Tables 4 and 5. The results show performance scores of the population through 5000 steps with interventions occurring at time step 1000. Table 4 shows performance scores for the group level intervention test case: social influence, cultural influence and the hybrid in the second, third and fourth columns respectively. Table 5 shows corresponding performance scores for the population level intervention test case.

It can be observed in Table 4 that after intervention population performance score increase to $0.87,0.82$ and 0.85 for social, cultural and hybrid influence. However, corresponding scores in Table 5 show a performance score increase to $0.54,0.58$ and 0.65 . This suggests that group level intervention outperforms population level intervention regardless of whether agents are affected by community beliefs. The second column of Table 5 shows lower performance scores for social influence than corresponding scores for cultural and hybrid influence. Although interventions occurred at population level, with social influence, agents' decisions depend on their group level encounters. As a result these agents do not benefit directly from population level intervention. Interestingly, interventions at group level are able to cause an emergence of better performance at the population level. It should be noted that agents targeted for intervention do not necessarily learn the correct knowledge perfectly since each agent's reception of the knowledge depends on its respective random learning rate. All results suggest that targeting the most influential members of a group or population for intervention can help spread optimal restraint use knowledge. 


\begin{tabular}{ccccc}
\hline Time Step & $\begin{array}{c}\text { Mean Distinct } \\
\text { Selection Patterns }\end{array}$ & $\begin{array}{c}\text { Mean Distinct } \\
\text { Use Patterns }\end{array}$ & $\begin{array}{c}\text { Mean Dominant } \\
\text { Selection Pattern }\end{array}$ & $\begin{array}{c}\text { Mean Dominant } \\
\text { Use Pattern }\end{array}$ \\
\hline 1 & 6.83 & 7.50 & 0.14 & 0.12 \\
100 & 5.17 & 4.83 & 0.33 & 0.38 \\
500 & 3.67 & 3.33 & 0.53 & 0.54 \\
1000 & 3.17 & 2.67 & 0.66 & 0.62 \\
5000 & 2.83 & 1.83 & 0.69 & 0.78 \\
\hline
\end{tabular}

Table. 1. Norm emergence of patterns of restraint selection and use with social influence, extracted from group belief spaces for 50 agents randomly organized in 6 groups.

Social Influence

Cultural Influence

\begin{tabular}{ccccc}
\hline Time Step & $\begin{array}{c}\text { Distinct \# of } \\
\text { Selection Patterns }\end{array}$ & $\begin{array}{c}\text { Distinct \# of } \\
\text { Use Patterns }\end{array}$ & $\begin{array}{c}\text { Distinct \# of } \\
\text { Selection Patterns }\end{array}$ & $\begin{array}{c}\text { Distinct \# of Use } \\
\text { Patterns }\end{array}$ \\
\hline 1 & 25 & 37 & 25 & 35 \\
100 & 20 & 26 & 15 & 24 \\
500 & 17 & 20 & 14 & 17 \\
1000 & 12 & 10 & 13 & 16 \\
5000 & 11 & 9 & 12 & 13 \\
\hline
\end{tabular}

Table. 2. Norm emergence of patterns of restraint selection and use with social and cultural influence, extracted from population belief space for 50 agents randomly organized in 6 groups.

\begin{tabular}{ccc}
\hline Time Step & $\begin{array}{c}\text { Distinct \# of } \\
\text { Selection } \\
\text { Patterns }\end{array}$ & $\begin{array}{c}\text { Distinct \# of } \\
\text { Use Patterns }\end{array}$ \\
\hline 1 & 25 & 37 \\
100 & 19 & 25 \\
500 & 17 & 22 \\
1000 & 17 & 20 \\
5000 & 12 & 17 \\
\hline
\end{tabular}

Table. 3. Norm emergence of patterns of restraint selection and use with a hybrid of social and cultural influence, extracted from population belief space for 50 agents randomly organized in 6 groups.

\begin{tabular}{cccc}
\hline $\begin{array}{c}\text { Time } \\
\text { Step }\end{array}$ & $\begin{array}{c}\text { Social } \\
\text { Pop. } \\
\text { Score }\end{array}$ & $\begin{array}{c}\text { Cultural } \\
\text { Pop. } \\
\text { Score }\end{array}$ & $\begin{array}{c}\text { Hybrid } \\
\text { Pop. Score }\end{array}$ \\
\hline 1 & 0.50 & 0.49 & 0.51 \\
100 & 0.50 & 0.50 & 0.44 \\
500 & 0.51 & 0.44 & 0.55 \\
1000 & 0.58 & 0.45 & 0.58 \\
2000 & 0.84 & 0.80 & 0.87 \\
5000 & 0.87 & 0.82 & 0.85 \\
\hline
\end{tabular}

Table. 4. Population performance for group level interventions applied at time step 1000 with social, cultural and hybrid influence, extracted from population belief space for 50 agents randomly organized in 6 groups.

\begin{tabular}{cccc}
\hline $\begin{array}{c}\text { Time } \\
\text { Step }\end{array}$ & $\begin{array}{c}\text { Social } \\
\text { Pop. } \\
\text { Score }\end{array}$ & $\begin{array}{c}\text { Cultural } \\
\text { Pop. } \\
\text { Score }\end{array}$ & $\begin{array}{c}\text { Hybrid } \\
\text { Pop. Score }\end{array}$ \\
\hline 1 & 0.49 & 0.50 & 0.47 \\
100 & 0.48 & 0.51 & 0.43 \\
500 & 0.47 & 0.52 & 0.49 \\
1000 & 0.48 & 0.58 & 0.44 \\
2000 & 0.53 & 0.56 & 0.62 \\
5000 & 0.54 & 0.58 & 0.65 \\
\hline
\end{tabular}

Table. 5. Population performance for population level interventions applied at time step 1000 with social, cultural and hybrid influence, extracted from population belief space for 50 agents randomly organized in 6 groups.

\section{Conclusions and Future Work}

In this study we provided a multi-agent simulation model to study interventions applied to emergent restraint misuse within groups of agents in the presence of social and cultural influences. Using aspects of a previously implemented model of artifact capabilities restraints are defined as artifacts with agents capable for their use. Randomly assigned influence rates are used during repeated encounters between group members to allow more influential agents to alter the knowledge of 
less influential ones. Cultural influence is implemented using a cultural algorithm and used to simulate agents affected by beliefs in their community. A hybrid form of influence in which agents are randomly affected either social or culturally is also investigated. While one series of experiments investigates the emergence of patterns of restraint selection and use, the other series of experiments investigates interventions at group and population level. At group level, the most influential member of each group is targeted for intervention and at population level the top $4 \%$ influential members of the population at large are targeted. Agent learning rates determine the probability to which they accept each piece of new knowledge and agent retention rates determine whether agents allow their knowledge to be altered at all. Results obtained from the conducted experiments confirm that particular patterns of restraint selection and use emerge over time. They suggest that it may not be necessary to target an entire population for intervention if influential members of the community can be identified and targeted instead. Additionally it was observed that interventions targeted at specific group members performed better than interventions targeted at influential members of the population at large. Although results sometimes showed a resistance in the system due to cultural influence, interventions seemed successful regardless of social, cultural or the hybrid form of influence tested.

In future work it may be useful to implement a social network in addition to groups. This might be a better reflection of the relationships between individuals that might not always exist in closed groups. In the current study altered agent knowledge is retained at each time step. It might be useful to support an agent making a decision without permanently altering its underlying knowledge. Another possible future direction is investigating what happens when agents leave a group with a particular norm and migrate to a group with a different norm, taking their capability for restraints with them.

\section{Acknowledgements}

This work has been supported by a grant from Auto21 and partial grants from CIHR and NSERC Discovery.

\section{References}

[ACAY, D.L. et al. 2008]

[BLAIR, J. et al. 2008]

[EBEL, B. et al. 2003]

[BROWN, J. et al. 2006]

[BRUCE, B.S. et al. 2011]

[BRUCKNER, R. et al. BRUCKNER, Richard, Rocker, J. Car safety. Pediatrics in Review 30

ACAY, Daghan L, Tilder, G., Sonenberg, L. Extending agent capabilities: tools vs agents. Proceedings of the IEEE/WIC/ACM International Conference on Web Intelligence and Intelligent Agent Technology (2008), pp. 259-265.

BLAIR, John, Perdios, A., Babul, S., Young, K., Beckles, J., Pike, I., Cripton, P., Sasges, D., Mulpuri, K., Desapriya, E. The appropriate and inappropriate use of child restraint seats in Manitoba. International Journal of Injury Control and Safety Promotion 15(3) (2008), pp. 151-156.

EBEL, Beth, Koepsell, T, Bennett, E., Rivara, F. Use of child booster seats in motor vehicles following a community campaign: a controlled trial. Journal of the American Medical Association 289 (2003), pp. 879-884.

BROWN, Julie, McCaskill, M.E., Henderson, M., Bilston, L.E. Serious injury is associated with suboptimal restraint use in child motor vehicle occupants. Journal of Paediatrics 42 (2006), pp. 345-349.

BRUCE, Beth, Snowdon, A.W., Cunningham, C., Cramm, C.L., Whittle, K., Correale, H., Barwick, M., Piotrowski, C., Warda, L., Harold, J. Predicting parents' use of booster seats. Injury Prevention 17(5) (2011), pp. 313-318. 
2009]

[BULGER, E.M. et al. 2008]

[CUNNINGHAM, C. et al. 2011]

[FELDMAN, D.C. et al. 1984]

[HOWARD, A. 2002]

[HOWARD, A. et al. 2004]

[KOBTI, Z. et al. 2006]

[MOKOM, F. et al. 2011]

[MOKOM, F. et al. 2013]

[OPP, K.D. et al. 2001]

[PEDEN, M. et al. 2004]

[PEDEN, M. et al. 2008]

[RANGEL, S. et al. 2008]

[REYNOLDS, R. et al. 2004]

[SAVARIMUTHU, B.T.R. et al. 2011]

[SEN, S. et al. 2007]

[SNOWDON, A. et al. 2009]
(2009), pp. 463-469.

BULGER, Eileen M, Kaufman, R., Mock, C. Childhood crash injury patterns associated with restraint misuse: implications for field triage. Prehospital and Disaster Medicine 23(1) (2008), pp. 9-15.

CUNNINGHAM, Charles E., Bruce, B.S., Snowdon, A.W., Chen, Y., Kolga, C., Piotrowski, C., Warda, L., Correale, H., Clark, E., Barwick, M. Modeling improvements in booster seat use: a discrete choice conjoint experiment. Accident Analysis and Prevention 43 (2011), pp. 1999-2009.

FELDMAN, Daniel, C. The development and enforcement of group norms. The Academy of Management Review 9(1) (1984), pp. 47-53.

HOWARD, Andrew, Automobile restraints for children: a review for clinicians. Canadian Medical Association Journal 167 (2002), pp. 769-773.

HOWARD, Andrew, Snowdon, A., MacArthur, C. Removing barriers to booster seat use in canada. Pediatrics \& Child Health 9 (2004), pp. 295-297. KOBTI, Ziad, Snowdon, A., Rahaman, S., Dunlop, T., Kent, R. A cultural algorithm to guide driver learning in applying child vehicle safety restraint. Proceedings of the IEEE Congress on Evolutionary Computation (2006), pp. 1111-1118.

MOKOM, Felicitas, Kobti, Z. Evolution of artifact capabilities. Proceedings of the IEEE Congress on Evolutionary Computation (2011), pp. 476-483.

MOKOM, Felicitas, Kobti, Z. Suboptimal restraint use as an emergent norm via social influence. Distributed Computing and Artificial Intelligence (2013), pp. 545-552.

OPP, Karl-Dieter. How do norms emerge? An outline $f$ a theory. Mind \& Society (2001), pp. 101-128.

PEDEN, Margie, Scurfield, R., Sleet, D., Mohan, D., Hyder, A.A., Jarawan, E., Mathers, C., eds. World report on road traffic injury prevention. World Health Organization (2004).

PEDEN, Margie, Oyegbite, K., Ozanne-Smith, J., Hyder, A.A., Branche, C., Rahman, A.F., Rivara, F., Bartolomeos, K., eds. World report on child injury prevention. World Health Organization (2008).

RANGEL, Shawn J., Martin, C.A., Brown, R.L., Garcia, V.F., Falcone Jr, R.A. Alarming trends in the improper use of motor vehicle restraints in children: implications for public policy and the development of race-based strategies for improving compliance. Journal of Pediatric Surgery 43 (2008), pp. 200-207.

REYNOLDS, Robert G, Peng, B. Cultural algorithms: modeling of how cultures learn to solve problems. Proceedings of the $16^{\text {th }}$ IEEE International Conference on Tools with Artificial Intelligence (2004), pp. 166-172.

SAVARIMUTHU, Bastin T.R., Cranefield, S. Norm creation, spreading and emergence: a survey of simulation models of norms in multi-agent systems. Multiagent and Grid Systems 7(1) (2011), pp. 21-54.

SEN, Sandip, Airiau, S. Emergence of norms through social learning. Proceedings of the $20^{\text {th }}$ International Joint Conference on Artificial Intelligence (2007), pp. 1507-1512.

SNOWDON, Anne, Hussein, A., Kolga, C., Bruce, B., Boase, P., PurcStevenson, R., Howard, A. Are we there yet? Canada's progress towards achieving road safety vision 2010 for children travelling in vehicles. International Journal of Injury Control and Safety Promotion 16(4) (2009), pp. 231-237. 